\title{
Experimental investigation on the effect of surface electric field in the growth of tungsten nano-tendril morphology due to low energy helium irradiation
}

\author{
K.B. Woller*, D.G. Whyte, G.M. Wright, and D. Brunner \\ MIT Plasma Science and Fusion Center, 175 Albany St., Cambridge, MA, USA, 02139
}

\begin{abstract}
:
The mechanisms responsible for and controlling the growth of tungsten nano-tendrils (or “fuzz") under low-energy helium plasma exposure remain unclear. For the first time in nanotendril experiments, the plasma sheath-produced electric field and the helium (He) ion energy have been decoupled, showing that the sheath electric field has little impact on nano-tendril growth, eliminating a possible cause for tendril growth. The well-established necessary growth conditions for $\mathrm{W}$ fuzz were maintained with $\mathrm{He}$ ion flux density $\Gamma_{\mathrm{He}}>10^{21} \mathrm{He} \mathrm{m}^{-2} \mathrm{~s}^{-1}$, surface temperature $\mathrm{T}_{\mathrm{s}}=1273 \mathrm{~K}, \mathrm{He}$ ion energy $\mathrm{E}_{\mathrm{He}}=64 \mathrm{eV}$, and $\mathrm{He}$ ion fluence $\Phi_{\mathrm{He}}>10^{24}$ $\mathrm{He}^{-2}$. A grid is situated between the tungsten sample and plasma, with the grid and sample potentials independently controlled in order to control the electric field at the surface of the sample while maintaining the same incident He ion energy to the surface. A calculation of the potential profile in the drift space between the grid and sample was used to account for space charge and calculate the electric field at the surface of the sample. Tungsten fuzz formed at all electric fields tested, even near zero electric field. Also, the depth of the resulting W fuzz layer was unaltered by the electric field when compared to the calculated depth determined from an empirical growth model. The conclusion is that the sheath electric field is not necessary to cause the changes in surface morphology.
\end{abstract}

PACS: 52.40.Hf, 82.80.Yc, 81.07-b, 81.05.Je

*Corresponding author address: MIT Plasma Science and Fusion Center, 175 Albany St., NW17-210, Cambridge, MA, USA, 02139

*Corresponding author e-mail: kbwoller@mit.edu 


\section{Introduction \& Motivation}

When tungsten $(\mathrm{W})$ surfaces at high temperatures $\left(0.25<\mathrm{T}_{\mathrm{H}}<0.45\right.$, where $\mathrm{T}_{\mathrm{H}}=\mathrm{T}_{\mathrm{S}} / \mathrm{T}_{\mathrm{M}}$ is the homologous temperature) are subjected to low energy helium (He) plasma below the sputter threshold for He bombardment of W, W nano-tendrils form on the surface. With continued exposure, the nano-tendrils increase in length and intertwine, forming an entangled layer of W nano-tendrils, referred to as W "fuzz." W fuzz is under investigation for the effects it may have on $\mathrm{W}$ plasma-facing components in fusion energy devices. It also provides a striking example of metallic surface reorganization with low-energy ion bombardment in a case where the incident ion species is highly insoluble in the metal. The surface morphology was discovered in linear plasma devices [1], but has since been observed in toroidal confinement devices [2] and ion beam experiments [3]. Factors influencing the W fuzz growth, determined through empirical studies, include the incident He ion energy, surface temperature, He ion flux density, and He ion fluence. There are thresholds in ion energy and surface temperature that are necessary for fuzz growth. For ion energy, the lower threshold ranges from 12 to $30 \mathrm{eV}$, depending on the experiment [4-6], and may depend on varying sample grade or plasma conditions, as well as surface temperature. An upper and lower bound for surface temperature sets a window for observation of nano-tendrils. W fuzz does not appear until the surface temperature is $900 \mathrm{~K}$. Below this temperature, pits and blisters appear in the surface. As the temperature increases, the features of the surface morphology increase in linear size $[7,8]$ until the surface becomes covered in large $(>10 \mu \mathrm{m})$ voids and structures above $2000 \mathrm{~K}$. If the temperature is raised high enough, the surface recrystallizes and nanostructuring ceases. Some physical models have been published, such as [9] and [10], which describe $\mathrm{W}$ fuzz growth as the surface "swelling and digging" as a result of He bubbles coalescing and rupturing. Other models, such as [11] and [12], suggest 
that enhanced surface diffusion of the $\mathrm{W}$ allows the surface to relax around the stress imposed by high pressure He bubbles; bubbles produced by the insolubility of $\mathrm{He}$ in W. However, through measurements we have previously made of the He concentration during $\mathrm{W}$ fuzz growth [13], it is known that the He bubbles do not possess the necessary He pressure to overcome the strength of the $\mathrm{W}$ nor does the surface have a high enough He concentration to effectively lower the surface energy of W. Given the extreme environment that results in W fuzz growth, there are other possible mechanisms that could lead to nano-tendril growth.

The majority of experiments involving $\mathrm{W}$ fuzz growth use low temperature plasma $\left(\mathrm{T}_{\mathrm{e}}<\right.$ $10 \mathrm{eV}$ ) as the source of the He ions. He ions are extracted from the plasma through the plasma sheath to a biased $\mathrm{W}$ sample. In most cases, the potential drop across the plasma sheath is on the order of $10 \mathrm{~V}$, but the thickness of the plasma sheath is $10^{-5}-10^{-4} \mathrm{~m}$ in linear plasma devices and tokamak divertor plasma environments. Therefore, there is an electric field $10^{5}-10^{6} \mathrm{~V} \mathrm{~m}^{-1}$ perpendicular to the solid surface. Surface structures due to thermal grooving and ion bombardment cause non-ideal surfaces that can further enhance the local electric field due to the plasma sheath by another two orders of magnitude [14,15]. Research in field ion microscopy (FIM), in which a high aspect ratio $\mathrm{W}$ probe is biased to a few kilovolts, shows that $\mathrm{W}$ electromigration, as a result of the large electric field, effectively "builds up" the probe tip [16,17]. Probe tip build up might be similar to how W nano-tendrils in W fuzz grow, but the electric field in FIM experiments is orders of magnitude larger than that due to a plasma sheath. In addition to the large probe bias, FIM experiments use He as an imaging gas. "Microprotrusions" with radii similar to the nano-tendrils of $\mathrm{W}$ fuzz are observed to grow on probe tips, which lead to arcing and vacuum breakdown. The growth of these "microprotrusions" is attributed to surface diffusion activated by He ion bombardment in the presence of large electric fields [18]. Even though plasma based W fuzz growth experiments have a lower electric field than in FIM, the plasma devices have a much larger 
ion flux density incident on the surface. Thus, there may be a synergistic effect of the sheath electric field and ion bombardment that results in $\mathrm{W}$ fuzz growth.

Recently, single W nano-tendrils were discovered in the DIONISOS plasma exposure chamber [19] under similar He irradiation conditions as W fuzz. The stand-alone W nanotendrils are similar in size to the nano-tendrils of $\mathrm{W}$ fuzz, but are observed freestanding without neighboring nano-tendrils for support. The similarity in aspect ratio of these solitary W nano-tendrils to other metal whiskers [20-22] studied since the mid-20th century suggests W nano-tendril and fuzz growth could be based on similar principles as metal whisker growth. Whisker growth is another example of dramatic surface modifications, and so it is natural to consider the possibility of a link between whiskers and fuzz. A model for metal whisker growth recently published [23] suggests that large electric fields induced by surface inhomogeneity could cause whisker nucleation and enhance whisker growth. Applied electric fields from the plasma sheath could play a role in initiating or enhancing the whisker/nanotendril growth process that develops into $\mathrm{W}$ fuzz. It is a combination of these observations that lead us to investigate the possible role of the sheath electric field in the nucleation and/or growth of W fuzz.

The electric field could affect $\mathrm{W}$ fuzz growth in two ways. First, the electric field polarization energy could affect the nucleation mechanism of nano-tendrils. As it is discussed in [23], the slender shape of a nano-scale metal whisker can possess electric field polarization energy due to the electric field within the metal whisker. The nucleation barrier to nucleate such metal whiskers is decreased when an electric field is present at the surface. The nucleation barrier derived by Karpov takes into consideration the surface electric fields inherent to metals just by virtue of surface inhomogeneity, which actually serves as the dominant source of polarization energy in his model. The order of this inherent electric field is $10^{8} \mathrm{~V} \mathrm{~m}^{-1}$ for materials similar to $\mathrm{W}$ [24] as determined by the spatial variation of the 
surface electric potential. Since the electric potential variation is inherent to the material, we cannot diminish the effect it may have in this experiment when coupled with ion bombardment as in FIM experiments. However, the electric potential variation alone would also be present during the high temperature annealing of samples, and we have not observed nano-tendril or whisker growth after a 1 hour anneal at $1273 \mathrm{~K}$.

The addition of an applied electric field via the plasma sheath decreases the nucleation barrier further. Thus, by decreasing the applied electric field, the nucleation density of metal whiskers on the surface would decrease. The decreased nucleation density would require a larger incubation fluence for nano-tendril growth. Therefore, the effect of decreasing the sheath electric field on nano-tendril nucleation would be observed through modifications to the incubation fluence.

Second, the electric polarization energy could also provide additional stimulus for nanotendril growth. The growth rate of metal whiskers in an external electric field is proportional to the polarization energy [23], $\mathbb{E}=-\varepsilon \alpha E^{2}$, where $\varepsilon$ is the permittivity, $\alpha$ is the polarizability, and $E$ is the electric field strength. Therefore, by decreasing the sheath electric field, one would expect the nano-tendril and $\mathrm{W}$ fuzz layer growth rate to decrease as well. Based on these proposed mechanisms, the overall effect of decreasing the sheath electric field would be to decrease $\mathrm{W}$ fuzz growth, due to increased incubation fluence and decreased growth rate after incubation.

\section{Experimental Materials and Methods}

\subsection{Growing tungsten Fuzz}

The experiment was conducted in the DIONISOS plasma exposure chamber [25] using continuous He plasma. First, a sample without a grid to control the electric field was grown to establish a control case that had the necessary conditions for fuzz growth. The subsequent exposures testing the effect of electric field would be conducted to match the exposure 
conditions of the control case as closely as possible. W samples were $99.95 \%$ pure PLANSEE $\mathrm{W}$ disks that were $1 \mathrm{~mm}$ thick and $25 \mathrm{~mm}$ in diameter. The surfaces were mechanically polished and then electropolished using a $4 \%$ sodium hydroxide solution kept at $0{ }^{\circ} \mathrm{C}$. The control $\mathrm{W}$ sample was mounted onto a ceramic heater that maintains the sample temperature using feedback from a thermocouple mounted inside the heater. The heater was taken to $1273 \mathrm{~K}$. At this temperature, the heat flux from the plasma when the sample is biased to $-50 \mathrm{~V}$ is comparable to the thermal radiation of polished $\mathrm{W}$. At the beginning of the exposure, the temperature of the surface of the $\mathrm{W}$ sample is $1273 \mathrm{~K}$. As the surface morphology changes to $\mathrm{W}$ fuzz, the emissivity increases to 1 [26], and the surface temperature is calculated to be $20 \mathrm{~K}$ lower than the heater temperature. Helium plasma was generated with an electron density of $6 \times 10^{17} \mathrm{~m}^{-3}$ and an electron temperature of $2.8 \mathrm{eV}$ as determined from a double Langmuir probe. This results in a He flux density of $3 \times 10^{21} \mathrm{He} \mathrm{m}^{-}$ ${ }^{2} \mathrm{~s}^{-1}$, which is in the range for fuzz growth $\left(>10^{20} \mathrm{He} \mathrm{m}^{-2} \mathrm{~s}^{-1}\right)$ [27]. The plasma potential was estimated from an uncompensated Langmuir probe to be $15 \mathrm{~V}$. The plasma potential measurement was further corroborated by a four-gridded retarding field energy analyzer, which measured the peak in He ion energy to be $14 \mathrm{eV}$ while the plasma facing grid was floating. The sample was biased with an additional $-50 \mathrm{~V}$ to bring the final $\mathrm{He}$ ion incident energy to $64 \mathrm{eV}$. The sample was then irradiated in the plasma to a fluence of $7.5 \times 10^{24} \mathrm{He}$ $\mathrm{m}^{-2}$, which is greater than the observed incubation fluence for W fuzz with ions of this energy $[28]$.

\subsection{Separating ion energy from surface electric field}

At the interface between the plasma and the sample surface, an electric field is naturally generated. The magnitude of the electric field, $|E|=|d \phi / d x|$, as determined from Poisson's equation with conservation of energy and flux [29], is

$$
|E|=2\left(\frac{J_{i}}{\epsilon_{0}} \sqrt{\frac{m_{i}}{2 e}}\right)^{1 / 2}\left(V_{p}-V_{\text {bias }}\right)^{1 / 4} \text {. }
$$


where $J_{i}$ is the ion current density entering the sheath that satisfies the Bohm criterion, $\epsilon_{0}$ is the permittivity of free space, $m_{i}$ is the mass of the ions, $e$ is the elementary charge, and $V_{p}-V_{\text {bias }}$ is the potential difference between the plasma and the sample surface.

The electric field at the surface of a sample exposed directly to the plasma described in section 2.1 with a measured current density of $487 \mathrm{~A} \mathrm{~m}^{-2}$, plasma potential of $14 \mathrm{~V}$, and a sample bias of $-50 \mathrm{~V}$ is $5 \times 10^{5} \mathrm{~V} \mathrm{~m}^{-1}$. To gain control over the electric field while maintaining control over the He ion energy, a grid was situated between the plasma and the sample. The grid wire spacing was chosen to adequately shield out the influence of the plasma sheath from the sample surface. The characteristic length to do so is proportional to the Debye length,

$$
\lambda_{\mathrm{D}}=\sqrt{\frac{\varepsilon_{0} \mathrm{kT}_{\mathrm{e}}}{\mathrm{e}^{2} \mathrm{n}_{\mathrm{e}}}}
$$

For these plasma conditions, the Debye length is $14 \mu \mathrm{m}$. To keep the plasma from leaking into the drift space between the grid and sample, the wire spacing of the grid has to have a maximum dimension that is only 2 - 4 Debye lengths [30]. The gap size of the grid used in this experiment is $40 \mu \mathrm{m}$, or approximately $3 \lambda_{\mathrm{D}}$, so the criterion to shield out the plasma from the sample is met for a floating grid. Additionally, in our case, the grid is biased negatively, which extends the sheath and improves shielding. To test the plasma shielding capabilities of the grid, we placed a $-50 \mathrm{~V}$ bias potential on the grid, and only ion current was measured on the sample for a sample voltage sweep from $-100 \mathrm{~V}$ to $+100 \mathrm{~V}$, indicating that the plasma was adequately shielded out of the drift space since no electron current could be measured even for positive sample bias with $\mathrm{V}>\mathrm{T}_{\mathrm{e}}$.

The $25 \mu \mathrm{m}$ thick grid has a transparency of 0.56 and is mounted on a $150 \mu \mathrm{m}$ thick tantalum grid holder. The grid holder is mounted in front of the sample with $230 \mu \mathrm{m}$ thick alumina spacers between the grid holder and the sample. This creates a total drift space thickness of $380 \mu \mathrm{m}$. The lateral size of the alumina spacers is comparable to their thickness. 
Eight alumina spacers were positioned in a circular array far away from the interaction region at the center of the sample holder to minimize the thermal conduction from the sample to the molybdenum clamping ring, which held the grid holder and alumina spacers in place by compression. A schematic of the modified sample is shown in Fig. 1.

The grid diminishes the ion flux incident on the sample surface due to the finite transparency of the grid. For this experiment, the flux density was taken as the measured current to the sample through the grid divided by the surface area observed to have grown $\mathrm{W}$ fuzz after exposure. The flux density calculated from these measurements during the experiment was in the range $1.2-1.6 \times 10^{21} \mathrm{He} \mathrm{m}^{-2} \mathrm{~s}^{-1}$, which is similar to the flux density from the plasma multiplied by the transparency of the grid. The flux density and fluence measured in this way are used for the drift space potential profile calculations described in section 2.3 and for determining the expected W fuzz layer thickness described in section 2.4.

\subsection{Drift space potential profile}

If there were no source of charge in the drift space, then the electric field would simply be the difference in potential between the grid and sample divided by the drift space distance. However, since the drift space contains ions extracted from the plasma, a potential distribution within the gap is present due to net positive space charge, and a subsequent electric field would be generated at the surface of the sample. Again, by solving Poisson's equation, along with continuity and conservation of energy, the potential distribution of the drift space [31] and the electric field at the surface of the sample were calculated. The 2D/3D effects of the grid wires were not taken into account as the drift space is an order of magnitude larger than the grid wires and spacing. The alumina spacers are very far removed from the grid area so that they do not affect the potential in the gap of the $1 \mathrm{~mm}$ by $1 \mathrm{~mm}$ irradiation region. Also, the calculation assumes there is no other source of charge in the drift space due to ionization. This is justified since the ionization mean free path is approximately 
$10 \mathrm{~mm}$, which is 100 times the gap spacing. Also, the calculation assumes the ions are traversing the gap in one dimension perpendicular to the surface. There is some spread in the thermal ion velocity distribution, but the effect on the potential distribution is assumed to be secondary to the average speed. The drift space distance is substantially larger than the ionion collision mean free path, which is calculated to be $50 \mu \mathrm{m}$. Therefore, momentum transfer collisions between ions have to be considered because the ions can gain additional velocity spread through the gap. To measure the energy spread through the drift space, we used the grid and sample as a single grid energy analyzer by sweeping the voltage on the sample, which acts as the collector for the energy analyzer, and holding the grid bias fixed. This measurement gives an ion energy spread through to the collector of $10-12 \mathrm{eV}$, assuming that the ions are extracted from the plasma at room temperature. This energy spread through the drift space is not incorporated in the space charge calculation, but the error introduced by neglecting the energy spread through the drift space is second order to the potential distribution calculated using the average extraction ion energy $>50 \mathrm{eV}$ through the grid. The potential distribution was calculated under the above assumptions using the current density measured during the experiment. Shown in Fig. 2 are the potential profiles for grid biases of $50 \mathrm{~V},-80 \mathrm{~V}$, and $-90 \mathrm{~V}$, with the sample bias fixed at $-50 \mathrm{~V}$, thus holding the incident ion energy at $64 \mathrm{eV}$.

In the case where the grid and sample are both $-50 \mathrm{~V}$, the surface electric field due solely to space charge is approximately $10 \%$ of the sheath electric field that would be at the sample surface without the grid. The lowest surface electric field was $3 \%$ of the sheath electric field, which required the grid-to-sample potential difference to be $30 \mathrm{~V}$ for the gap spacing and current densities in this experiment (see Fig. 2).

\subsection{Comparison to open plasma tungsten fuzz growth}


In order to assess if changing the electric field affects the resulting fuzz layer, we compare the grid-grown fuzz to fuzz grown without the grid. However, due to small variations in plasma discharges, grid-sample mounting, and grid biasing, there are differences in the fluence for each exposure. The fuzz growth has been shown to follow diffusion-like growth in conditions similar to that used in this work [32]. Calculating the diffusion coefficient, D, at $1273 \mathrm{~K}$ using the pre-exponential factor of $9.9 \times 10^{-14} \mathrm{~m}^{2} \mathrm{~s}^{-1}$ and activation energy of $0.71 \mathrm{eV}$, interpolated from data at $1120 \mathrm{~K}$ and $1320 \mathrm{~K}$ from the work of Baldwin and Doerner [32], we can calculate based on this empirical formula, the expected thickness $\left(\mathrm{h}_{\text {Baldwin }}\right.$ ) of the resulting fuzz layers depending on the fluence, or exposure time of each exposure,

$$
\mathrm{h}_{\text {Baldwin }}=\sqrt{2 \cdot \mathrm{D}_{1273 \mathrm{~K}} \cdot \mathrm{t}} \text {. }
$$

The fuzz layer depth was also shown to depend on the He flux density [27]. In order to account for the lower flux density used here compared to those for the Baldwin formula, a correction factor was applied from the flux density scan performed by Baldwin and colleagues [27]. The $\mathrm{W}$ fuzz growth rate saturates at a He flux density of $10^{22} \mathrm{~m}^{-2} \mathrm{~s}^{-1}$. The flux density in this study is $1.2-3 \times 10^{21} \mathrm{~m}^{-2} \mathrm{~s}^{-1}$. The depth of the fuzz layer in [27] for a flux density similar to this work was $1 / 5$ that of the saturation fuzz layer depth. Thus, we calculate an expected fuzz layer depth in this work from empirical data as

$$
\mathrm{h}_{\mathrm{cal}}=\frac{1}{5} \cdot \sqrt{2 \cdot \mathrm{D}_{1273 \mathrm{~K}} \cdot \mathrm{t}}
$$

The fuzz layer depth was measured from cross section images made by Focused-Ion Beam (FIB) milling and compared to the empirical depth calculated with Eqn. (4).

\section{Results}

\subsection{Morphology Comparison}

The morphologies of $\mathrm{W}$ fuzz grown with and without the grid setup are shown in Fig. 3a and $3 b$, respectively, from a view normal to the surface. The electric field calculated for the two cases pictured are $5 \times 10^{5} \mathrm{~V} \mathrm{~m}^{-1}$ for the no grid sample and $1.5 \times 10^{4} \mathrm{~V} \mathrm{~m}^{-1}$ for the sample 
shielded by a grid biased at $-80 \mathrm{~V}$. The characteristic size of the nano-tendrils has not been altered compared to the control case, in agreement with the experimental evidence that the surface temperature has a strong influence on tendril size during $\mathrm{W}$ fuzz growth at these ion energies $[7,8]$, either through surface tension or He bubble size. There is no fundamentally different nano-tendril morphology when the $\mathrm{W}$ sample is shielded behind a grid and is, therefore, not subjected to the plasma directly.

\subsection{Growth Rate Comparison}

When viewing this complex surface in the normal direction, it is unclear to what extent the sheath electric field played a role in the formation and growth of the fuzz. If the sheath electric field did play a role in the growth of $\mathrm{W}$ fuzz, one might expect the growth rate to be proportional to the square of the electric field through the electric polarization energy, as discussed in section 1. We have measured the depths of the fuzz layers grown with several low electric field situations. The ratio of the grown fuzz layers to the calculated empirical value versus the magnitude of the calculated electric field is plotted in Fig. 4. The thickness of the fuzz layer, even with very low electric fields, is independent of the surface electric field (see Fig. 3c and 3d). Thus, we can conclude that the sheath electric field of the plasma that is used to grow $\mathrm{W}$ fuzz does not factor into the resulting fuzz growth, in morphology or growth rate.

Additionally, a bias configuration was used that had a slightly retarding electric field at the surface of the sample, approximately $5 \%$ of the sheath electric field and is included in Fig. 4 as the point labelled "-90V". The low positive field polarization did not affect the resulting fuzz growth. As may be seen in Figs. 3 and 4, the fuzz layer and its growth are almost identical whether subjected directly to plasma or shielded; there is no drastic change in the growth of the fuzz layer.

\section{Discussion}


Although we conclude that the plasma sheath electric field does not factor into the growth of W fuzz, this experiment does not address the possibility suggested in section 1 that inherent variations in surface potential due to the minimization of the electrochemical potential can generate large electric fields that could induce whisker/nano-tendril nucleation when coupled with He ion bombardment or He implantation. The FIM experiments do see a broad surface diffusion mechanism that is activated with the applied bias, but the microprotrusions were observed to be activated with the ion bombardment. The possibility of He bubbles factoring into the FIM experiments were considered by Schwoebel in [18], but the conclusion was that at the higher temperatures of $1200 \mathrm{~K}$, similar to fuzz growth, the He bubbles could not nucleate and grow, unlike He plasma irradiated W, which readily develop He nano-bubble fields. The lower He flux and higher ion energy in the FIM experiments as compared to the plasma irradiation may be the reason for this difference in the bubble behavior. Even so, ion bombardment enhanced diffusion is worth pursuing as a possible mechanism to be tested in the development of W nano-tendrils.

Upon observing the solitary $\mathrm{W}$ nano-tendrils in DIONISOS [19] we have been investigating why those exposures did not produce common $\mathrm{W}$ fuzz, similarly as why we and others have been investigating why $\mathrm{W}$ fuzz itself exists. The inception of the experiment reported here was to test whether surface electric fields enabled W fuzz growth because of the ubiquity of the plasma sheath in the various observations of W fuzz growth. The null result of this experiment prompts us to continue to investigate these intriguing morphologies and their growth mechanisms. After this study was concluded, we used the grid-sample setup to measure the ion energy distribution (IED) under the plasma conditions that produce solitary and bundled W nano-tendrils. The IED measurement provided a credible lead and will be reported on elsewhere.

\section{Summary}


We have tested whether the sheath electric field plays a role in the nucleation or subsequent growth of the nano-tendril morphology that occurs when He plasma irradiates a W surface at elevated surface temperature. By implementing an appropriate grid between the $\mathrm{W}$ sample and the He plasma, we have decreased the electric field at the surface of the sample to $3 \%$ of the electric field that would be present at the sample from the plasma sheath.

The resulting $\mathrm{W}$ fuzz layers grown in this study, with direct exposure to the plasma, with a much lower electric field, and even with a low retarding electric field, are all similar in terms of their nano-tendril size and layer depth, when normalized to an empirical layer depth calculation. We conclude that the sheath electric field does not impact the nucleation process nor the growth rate of the nano-tendrils that form when He plasma irradiates $\mathrm{W}$ at elevated surface temperatures.

\section{Acknowledgements}

This work is supported by US DOE award DE-SC00-02060. This work made use of the MRSEC Shared Experimental Facilities at MIT, supported by the National Science Foundation under award number DMR-08-19762.

\section{References}

[1] S. Takamura, N. Ohno, D. Nishijima, S. Kajita, Formation of Nanostructured Tungsten with Arborescent Shape due to Helium Plasma Irradiation, Plasma Fusion Res. 1 (2006) 051-051. doi:10.1585/pfr.1.051.

[2] G.M. Wright, D. Brunner, M.J. Baldwin, R.P. Doerner, B. Labombard, B. Lipschultz, J.L. Terry, D.G. Whyte, Tungsten nano-tendril growth in the Alcator C-Mod divertor, Nucl. Fusion. 52 (2012) 042003. doi:10.1088/0029-5515/52/4/042003.

[3] F.W. Meyer, H. Hijazi, M.E. Bannister, P.S. Krstic, J. Dadras, H.M. Meyer, C.M.

Parish, He-ion and self-atom induced damage and surface-morphology changes of a hot W target, Phys. Scr. T159 (2014) 014029. doi:10.1088/0031-8949/2014/T159/014029. 
[4] S. Kajita, W. Sakaguchi, N. Ohno, N. Yoshida, T. Saeki, Formation process of tungsten nanostructure by the exposure to helium plasma under fusion relevant plasma conditions, Nucl. Fusion. 49 (2009) 095005. doi:10.1088/0029-5515/49/9/095005.

[5] M.J. Baldwin, T.C. Lynch, R.P. Doerner, J.H. Yu, Nanostructure formation on tungsten exposed to low-pressure rf helium plasmas: A study of ion energy threshold and early stage growth, J. Nucl. Mater. 415 (2011) S104-S107. doi:10.1016/j.jnucmat.2010.10.050.

[6] G. De Temmerman, K. Bystrov, J.J. Zielinski, M. Balden, G. Matern, C. Arnas, L. Marot, Nanostructuring of molybdenum and tungsten surfaces by low-energy helium ions, J. Vac. Sci. Technol. A Vacuum, Surfaces, Film. 30 (2012) 041306. doi:10.1116/1.4731196.

[7] W. Sakaguchi, S. Kajita, N. Ohno, M. Takagi, H. Kurishita, Formation Condition of Fiberform Nanostructured Tungsten by Helium Plasma Exposure, Plasma Fusion Res. 5 (2010) S1023-S1023. doi:10.1585/pfr.5.S1023.

[8] G.M. Wright, D. Brunner, M.J. Baldwin, K. Bystrov, R.P. Doerner, B. Labombard, B. Lipschultz, G. De Temmerman, J.L. Terry, D.G. Whyte, K.B. Woller, Comparison of tungsten nano-tendrils grown in Alcator C-Mod and linear plasma devices, J. Nucl. Mater. 438 (2013) S84-S89. doi:10.1016/j.jnucmat.2013.01.013.

[9] S. Kajita, N. Yoshida, N. Ohno, Y. Tsuji, Growth of multifractal tungsten nanostructure by He bubble induced directional swelling, New J. Phys. 17 (2015) 1-14. doi:10.1088/1367-2630/17/4/043038.

[10] a Lasa, S.K. Tähtinen, K. Nordlund, Loop punching and bubble rupture causing surface roughening - A model for W fuzz growth, EPL (Europhysics Lett. 105 (2014) 25002. doi:10.1209/0295-5075/105/25002.

[11] S.I. Krasheninnikov, Viscoelastic model of tungsten "fuzz" growth, Phys. Scr. T145 
(2011) 014040. doi:10.1088/0031-8949/2011/T145/014040.

[12] Y. V. Martynenko, M.Y. Nagel, Model of fuzz formation on a tungsten surface, Plasma-Surface Interact. 38 (2012) 996-999. doi:10.1134/S1063780X12110074.

[13] K.B. Woller, D.G. Whyte, G.M. Wright, Dynamic measurement of the helium concentration of evolving tungsten nanostructures using Elastic Recoil Detection during plasma exposure, J. Nucl. Mater. 463 (2015) 289-293. doi:10.1016/j.jnucmat.2014.11.126.

[14] W. Ahmed, E.S. Kooij, A. van Silfhout, B. Poelsema, Quantitative Analysis of Gold Nanorod Alignment after Electric Field-Assisted Deposition, Nano Lett. 9 (2009) 3786-3794. doi:10.1021/n1901968e.

[15] M. Nardone, V.G. Karpov, Nucleation of metals by strong electric fields, Appl. Phys. Lett. 100 (2012). doi:10.1063/1.3703611.

[16] J.P. Barbour, F.M. Charbonnier, W.W. Dolan, W.P. Dyke, E.E. Martin, J.K. Trolan, Determination of the surface tension and surface migration constants for tungsten, Phys. Rev. 117 (1960) 1452-1459. doi:10.1103/PhysRev.117.1452.

[17] P.C. Bettler, F.M. Charbonnier, Activation energy for the surface migration of tungsten in the presence of a high-electric field, Phys. Rev. 119 (1960) 85-93. doi:10.1103/PhysRev.119.85.

[18] P.R. Schwoebel, The epitaxial growth of microprotrusions on field emitter tips, J. Appl. Phys. 64 (1988) 2359. doi:10.1063/1.341666.

[19] K. Woller, D. Whyte, G. Wright, In Situ Elastic Recoil Detection Analysis of Tungsten Surfaces during ITER-like Helium Irradiation, in: 2015 IEEE 26th Symp. Fusion Eng., IEEE, Austin, Texas, 2015: pp. 1-6. doi:10.1109/SOFE.2015.7482349.

[20] S.S. Brenner, Growth and Properties of "Whiskers": Further research is needed to show why crystal filaments are many times as strong as large crystals, Science (80-. ). 128 
(1958) 569-575. doi:10.1126/science.128.3324.569.

[21] J.A. Brusse, G.J. Ewell, J.P. Siplon, Tin Whiskers : Attributes and Mitigation, 2002.

[22] G.T. Galyon, Annotated tin whisker bibliography and anthology, IEEE Trans. Electron. Packag. Manuf. 28 (2005) 94-122. doi:10.1109/TEPM.2005.847440.

[23] V.G. Karpov, Electrostatic theory of metal whiskers, Phys. Rev. Appl. 1 (2014) 1-13. doi:10.1103/PhysRevApplied.1.044001.

[24] J.B. Camp, T.W. Darling, R.E. Brown, Macroscopic variations of surface potentials of conductors, J. Appl. Phys. 69 (1991) 7126. doi:10.1063/1.347601.

[25] G.M. Wright, H. a Barnard, L. a Kesler, E.E. Peterson, P.W. Stahle, R.M. Sullivan, D.G. Whyte, K.B. Woller, An experiment on the dynamics of ion implantation and sputtering of surfaces, Rev. Sci. Instrum. 85 (2014) 023503. doi:10.1063/1.4861917.

[26] S. Kajita, T. Yokochi, N. Ohno, T. Kumano, Near Infrared Radiation from Heated Nanostructured Tungsten, Jpn. J. Appl. Phys. 51 (2012) 01AJ03. doi:10.1143/JJAP.51.01AJ03.

[27] M.J. Baldwin, R.P. Doerner, D. Nishijima, K. Tokunaga, Y. Ueda, The effects of high fluence mixed-species (deuterium, helium, beryllium) plasma interactions with tungsten, J. Nucl. Mater. 390-391 (2009) 886-890. doi:10.1016/j.jnucmat.2009.01.247.

[28] S. Kajita, N. Yoshida, R. Yoshihara, N. Ohno, M. Yamagiwa, TEM observation of the growth process of helium nanobubbles on tungsten: Nanostructure formation mechanism, J. Nucl. Mater. 418 (2011) 152-158. doi:10.1016/j.jnucmat.2011.06.026.

[29] M.A. Lieberman, A.J. Lichtenberg, Principles of Plasma Discharges and Materials Processing, 2nd ed., John Wiley \& Sons, 2005.

[30] R.A. Pitts, Retarding Field Energy Analysis for Ion Temperature Measurement in the Tokamak Edge, Contrib. to Plasma Phys. 36 (1996) 87-93. doi:10.1002/ctpp.19960360114. 
[31] G. Jaffé, On the Currents Carried by Electrons of Uniform Initial Velocity, Phys. Rev. 65 (1944) 91-98. doi:10.1103/PhysRev.65.91.

[32] M.J. Baldwin, R.P. Doerner, Helium induced nanoscopic morphology on tungsten under fusion relevant plasma conditions, Nucl. Fusion. 48 (2008) 035001. doi:10.1088/0029-5515/48/3/035001. 
Figure captions:

Fig. 1. Profile schematic of the modified sample with the grid mounted between the W surface and the He plasma. The detail of the interaction region between the grid and the sample surface is shown to scale in Fig. 2.

Fig. 2. Potential distributions with respect to ground calculated for the sheath potential drop and drift space for (a) no grid and three exposures with grid biases of (b) $-50 \mathrm{~V}$, (c) $-80 \mathrm{~V}$, and (d) $-90 \mathrm{~V}$. The sample had a bias of $-50 \mathrm{~V}$ and the plasma potential was measured to be $14 \mathrm{~V}$. The grid is spaced $3.8 \times 10^{-4} \mathrm{~m}$ from the sample surface located at $\mathrm{x}=0$.

Fig. 3. SEM micrographs showing the view of the control tungsten fuzz sample grown at $1273 \mathrm{~K}$ with $64 \mathrm{eV}$ ions at $3.0 \times 10^{21} \mathrm{He} \mathrm{m}^{-2} \mathrm{~s}^{-1}$ to a fluence of $7.5 \times 10^{24} \mathrm{He} \mathrm{m}^{-2}$ (a) normal to the surface and (c) in cross section. Also, the view of the W fuzz sample grown with the same plasma, but shielded by a grid with a bias of $-80 \mathrm{~V},(\mathrm{~b})$ normal to the surface and (d) in cross section. The flux density and fluence to the surface of the sample in the shielded case were $1.6 \times 10^{21} \mathrm{He} \mathrm{m}^{-2} \mathrm{~s}^{-1}$ and $6.9 \times 10^{24} \mathrm{He} \mathrm{m}^{-2}$, respectively.

Fig. 4. Fuzz layer thickness, $\mathrm{h}_{\mathrm{exp}}$, as measured by focused ion beam milling normalized to the fuzz layer thickness, $h_{\text {cal }}$, calculated using the empirical growth model Eqn. (4). The results are shown for the four biasing conditions of Fig. 2, corresponding to the data labels. The error bars denote the error in determining the layer thickness from cross section images. 
Fig. 1.

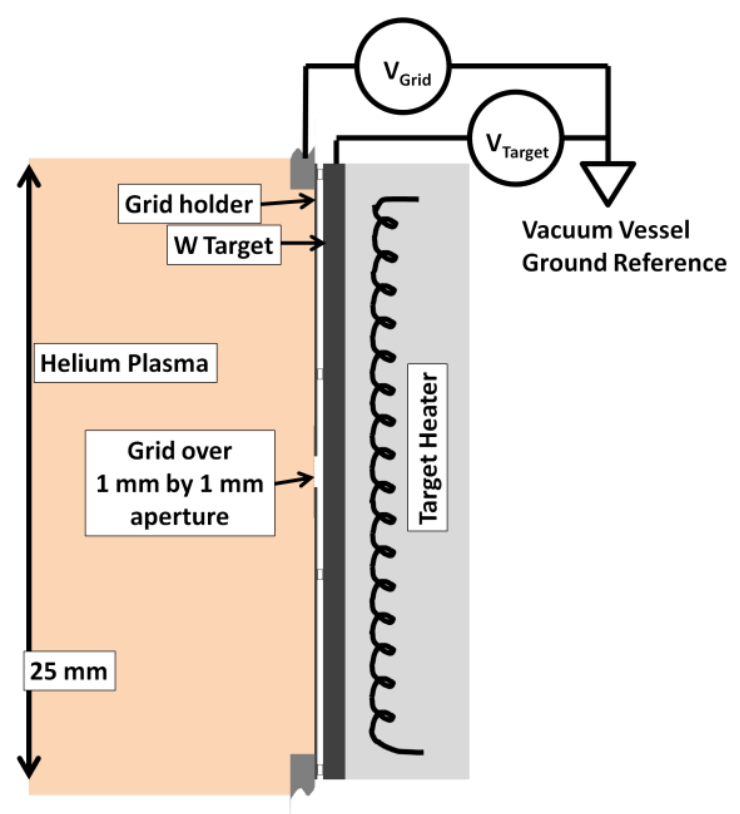


Fig. 2.

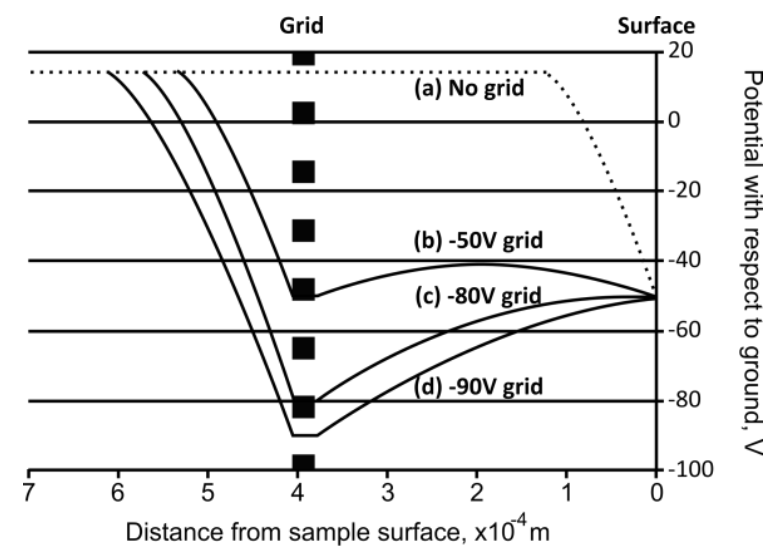


Fig. 3.
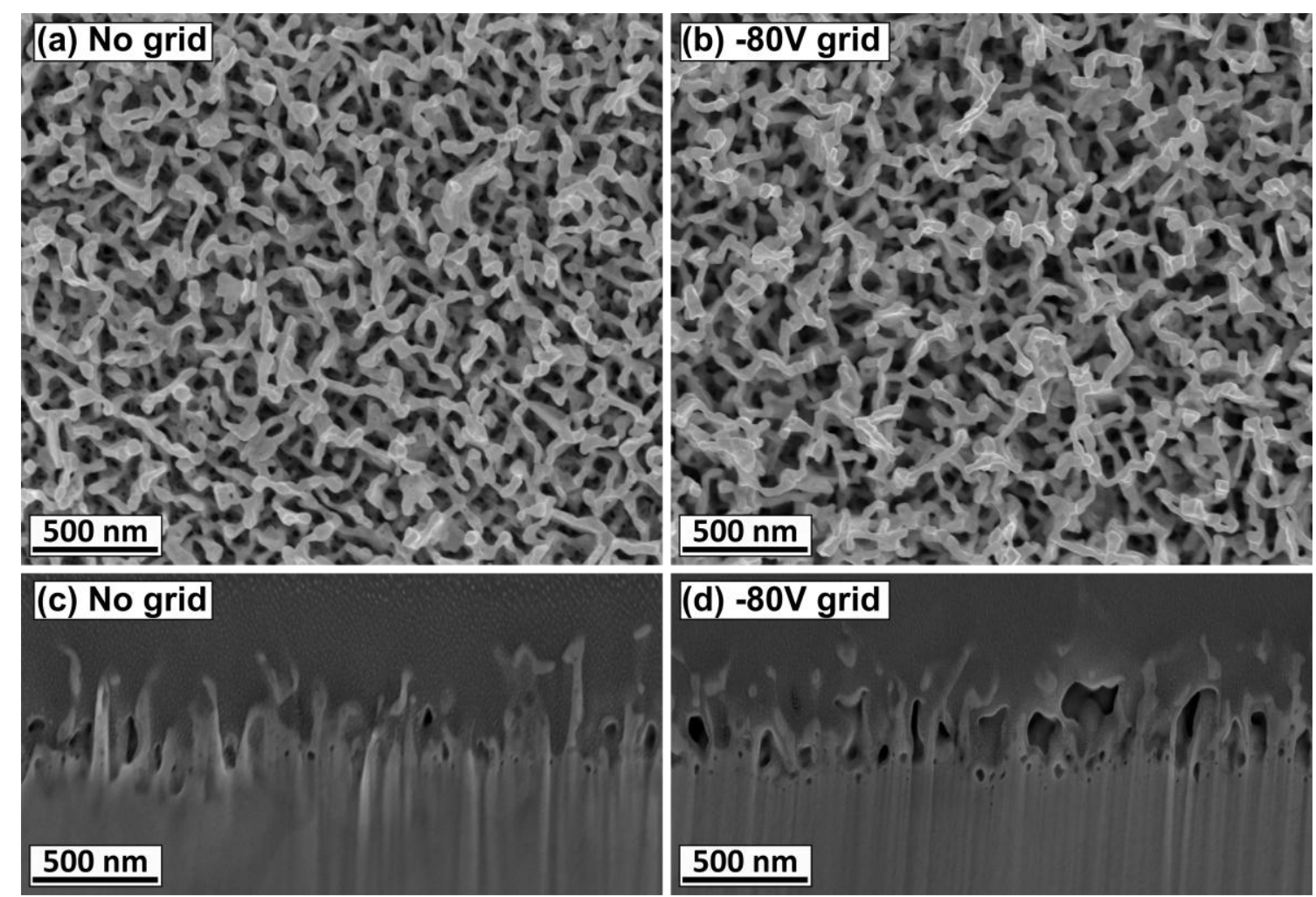
Fig. 4.

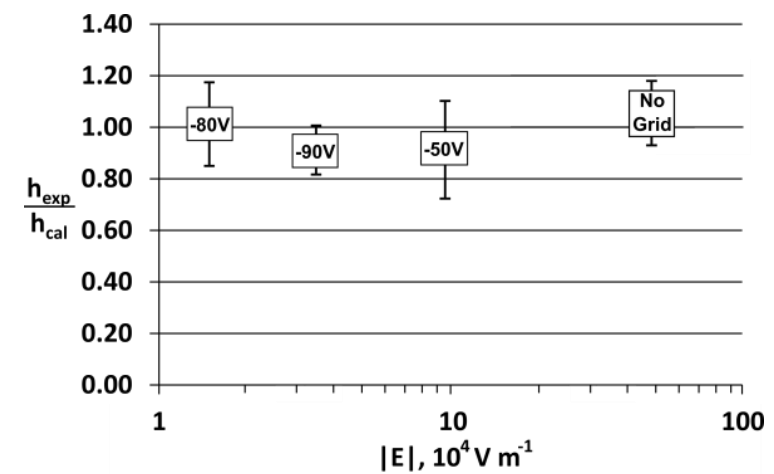

